\title{
The Atomic Mass Unit
}

\author{
BRIAN W. PETLEY
}

\begin{abstract}
This paper reviews the evolution and realization of the atomic mass unit and discusses prospects of replacing the prototype kilogram by an atomic mass standard.
\end{abstract}

\section{INTRODUCTION}

$T_{w}$ HE DIFFICULTIES of realizing the electrical units with an accuracy comparable to the precision achieved in maintaining a representation of the volt with the ac Josephson effect is fueling discussion of how the prototype kilogram might be replaced [1]. There is already an atomic mass unit $u$ (and an atomic mass constant $m_{u}$ ), and so it is appropriate to discuss the accuracy with which the atomic mass unit is presently realized in terms of the kilogram.

The Système International d'Unités (SI) is based on the concept of a coherent system of units [2] but the Conférence Générale des Poids et Mesures (CGPM) has recognized that certain "off-system" units would continue: such as the hour, day, and month. These recognized "offsystem" units also include the experimentally determined atomic mass unit and the electron volt (initially termed equivalent volt [3]). The atomic mass unit and the electron volt $(\mathrm{eV})$ are in widespread use in science and technology-in atomic energy, biology, chemistry, medicine, and in physics. The equivalence of mass and energy, via the Einstein equation $E=m c^{2}$, which involves the speed of light $c$, is so much a part of elementary particle physics that the masses of fundamental particles are commonly expressed in electronvolt $/ c^{2}$, or even in gigaelectronvolt!

\section{A Brief History of the Atomic Mass Unit}

The hydrogen atom was used by Dalton, U.K., in 1803 as a natural unit for the expression of atomic weights [4] and his unit was also adopted by Avogadro and Carrizaro (Italy). In 1818 Berzalo (Sweden) chose instead to use oxygen to the arbitrary base 100 for his atomic weight unit. Ostwald (Germany) pointed out in 1885 that more of the elements would have essentially integral numbers for their atomic weights if atomic weights were expressed in terms of a scale on which the mass of oxygen was 16 . The arguments as to which was the better of the two elements to use continued for many years, but the use of ${ }^{16} \mathrm{O}$ gradually prevailed. The International Union of Pure and

Manuscript received June 10,1988 .

The author is with the National Physical Laboratory, Teddington, Middlesex TW 11 OLW, U.K.

IEEE Log Number 8826183.
Applied Chemistry (IUPAC) set up the International Commission on Atomic Weights in 1920, which was charged to produce an internationally acceptable table of atomic weights, based on $\mathrm{O}=16$, at suitable intervals.

Although the first isotopes were discovered in 1912, the use of $O=16$ continued straightforwardly until 1929, when Giauque and Johnston announced their spectroscopic discovery of the isotopes of oxygen. This led to the adoption of two atomic weight scales [5]. That used by the chemists was based on the number 16 as representing the average mass of the oxygen atom in its natural state $\left({ }^{16} \mathrm{O}:{ }^{18} \mathrm{O}:{ }^{17} \mathrm{O}\right.$ in the ratio $\left.506: 1: 0.204\right)$, whereas the physicists considered the oxygen isotope ${ }^{16} \mathrm{O}$ as the basic unit on their scale. The dual system of units became increasingly inconvenient. For example, there were physical and chemical values for the Avogadro constant and the Faraday constants which differed by some $275(10)$ parts per million.

In 1960 the International Union of Pure and Applied Physics and IUPAC agreed [6] to adopt a unified scale which was based on the ${ }^{12} \mathrm{C}$ isotope. The symbol $u$ was adopted to represent the unified atomic mass unit defined as $1 / 12$ of the mass of the ${ }^{12} \mathrm{C}$ atom, that is,

$$
\begin{gathered}
1 \mathrm{u}=(1 / 12) m_{a}\left({ }^{12} \mathrm{C}\right), \quad m_{u}=(1 / 12) m_{a}\left({ }^{12} \mathrm{C}\right), \\
\text { and } m_{u}=1 \mathrm{u} .
\end{gathered}
$$

The symbol $M_{r}$ is used to represent relative molar mass as well as to denote relative atomic mass, $m_{a} / m_{u}$. The adoption of the unified atomic mass unit reduced the values on the earlier chemical scale by a factor 1.000043 , and the values on the old physical scale by a factor 1.000315 . The choice of suitable isotopes to denote the new mass unit was limited to those lying within 50 parts per million of the old scale of chemical atomic weights so that the extensive amount of numerical data in the chemical literature could remain essentially unchanged.

The quantity $m_{u}$ was initially termed the nuclidic mass unit. The agreement after the many years of debate was celebrated by denoting the unit by the symbol $u$, which was derived from unified-a word that is often omitted today. The unified mass unit is sometimes referred to by the word dalton (symbol Da). The use of the dalton is widespread in biology and biochemistry [7], where one might refer to a 180 -dalton glucose molecule or a $55-\mathrm{kDa}$ protein. However, this name for the atomic mass unit has not been approved by the CGPM. In any case the use in biology is far from the $10^{-9}$ precision achieved in modern mass spectrometry. 


\section{The Present Situation}

The physicists require an atomic mass scale on which to express the masses of nuclear isotopes, and the chemists require an atomic weight scale for expressing the relative masses of the elements containing isotopes in their natural relative abundances. From many points of view it remains convenient to distinguish between the two types of quantity by the terminology atomic mass and atomic weight, but this is the subject of a continuing debate. An important distinction is that whereas the atomic mass of an isotope is an invariant quantity, the atomic weight of an element is not a constant. Only 20 elements have only one isotope that occurs in nature and consequently the relative isotopic abundances of other elements can vary from one part of the earth to another and from one part of the universe to another. Most atomic weights are quoted to an accuracy of one digit in the fourth significant figure, but there are at least 32 elements where the variation is greater than this. Today the atomic masses of many isotopes are known to better than a part in $10^{8}$. There are some 280 stable or near stable atomic species and five or six times as many unstable ones. The evaluation of the best values of the atomic masses [8], [9] involves considering data from more than 2000 sources.

The atomic mass of an atom ${ }_{Z}^{Z+N} X, m_{a}(X)$, containing $Z$ electrons and protons and $N$ neutrons is given by

$\left.m_{a}(X)=\left[\left(Z m_{e}+Z m_{p}+N m_{n}\right)+\left(E_{n}+E_{e}\right) / c^{2}\right] / m_{u}\right]$

where $E_{n}$ is the nuclear binding energy and $E_{e}$ is the electron binding energy. The electron binding energy is very small for the lighter atoms although it may approach 0.1 percent of the binding energy of the heavier atoms. Aside from the stable isotopes, much of the recent research interest has been in deriving more accurate atomic masses for nuclei which are far from stability. This is because prediction of their nuclear properties provides stringent tests of the increasingly sophisticated models that have been developed during the last few years.

\section{The Realization of the Atomic Mass Unit}

The Avogadro constant $N_{A}$ relates the atomic mass unit and the kilogram via the molar mass of ${ }^{12} \mathrm{C}$ and hence also involves the mole, that is,

mass of ${ }^{12} \mathrm{C}$ atom

$=\left[\right.$ molar mass of $\left.{ }^{12} \mathrm{C}\right] /($ Avogadro constant $)$.

Also we have $m_{u}=M^{\ominus} / N_{A}$, where $M^{\ominus}$ [11] is the standard molar mass unit $=10^{-3} \mathrm{~kg} \cdot \mathrm{mol}^{-1}[10]$, and $m_{p} / m_{u}$ $=M(p) / M^{\theta}$, where $M(p)$ is the molar mass of the proton.

In the 1986 evaluation of the "best values" of the fundamental physical constants by Cohen and Taylor [10], the mass of the proton was derived from the evaluated unknowns: $\alpha$ and $K_{V}$ (where $K_{V}$ is the ratio of the as-maintained volt to the volt), together with such auxiliary constants as the Rydberg constant $R_{\infty}, E$ (the $1973 \mathrm{CCE}$ agreed value for the Josephson frequency to voltage quotient $2 e / h)$, and $m_{p} / m_{e}$, by the equation

$$
m_{p}=16 R_{\infty} K_{V}^{2}\left(m_{p} / m_{e}\right) /\left(\mu_{0} \alpha^{2} c^{2} E^{2}\right) .
$$

Our present knowledge of $m_{p}$, and hence $m_{u}$, therefore, depends either on the measurement of the Avogadro constant or on the experimental realizations of the electrical units: either directly via volt, ampere watt, and ohm, together with measurements pertaining to the fine-structure constant, as well as via the high-field measurements of the gyromagnetic ratio of the proton and the Faraday constant (see [11]).

The atomic mass of hydrogen can be derived from experimental measurements of mass doublets. The late Lincoln Smith measured [12] 14 distinct doublet combinations of the six masses: ${ }^{1} \mathrm{H},{ }^{2} \mathrm{H},{ }^{14} \mathrm{~N},{ }^{16} \mathrm{O},{ }^{35} \mathrm{Cl}$, and ${ }^{37} \mathrm{Cl}$. Direct mass comparisons with ${ }^{12} \mathrm{C}$ may also be made. Thus Smith used $\mathrm{C}_{9} \mathrm{H}_{22}^{+}-\mathrm{C}_{10} \mathrm{H}_{10}^{+}$, while Benson [13] used $\mathrm{C}_{11} \mathrm{H}_{22}^{+}-\mathrm{C}_{12} \mathrm{H}_{10}^{+}$. Given $m_{p}, m_{p} / m_{u}, m_{p} / m_{e}$, and the electron binding energy, one can obtain the mass of ${ }^{i} \mathrm{H}$ and $m_{u}$. Other atoms are used as secondary atomic mass standards, for example ${ }^{1} \mathrm{H},{ }^{2} \mathrm{H},{ }^{16} \mathrm{O},{ }^{14} \mathrm{~N},{ }^{13} \mathrm{C},{ }^{35} \mathrm{Cl}$, and ${ }^{37} \mathrm{Cl}$.

The CODATA 1986 recommended values of the fundamental physical constants [10] show that $m_{p} / m_{u}$ is known with a standard deviation uncertainty of 0.01 parts per million, and $m_{u}$ with an uncertainty of 0.6 part per million. It is evident from (2) that if we were to define the volt in terms of an agreed value for $E$ our knowledge of $m_{p}$ would be limited by our knowledge of the fine-structure constant. The latter is known at present with a fractional accuracy of $0.045 \times 10^{-6}$ and the corresponding imprecision of $m_{p}$ would be twice this. Thus although we would get to our atomic mass unit with ten times greater accuracy than at present, we would still have to use the atomic mass unit to avoid loss of accuracy in precision mass spectrometry.

\section{The Electron Volt and the Atomic Mass Unit}

Two sets of experimental data must be merged in order to derive recommended values: the atomic mass data from mass spectrometry, and the data concerning nuclear reaction energies [14]. The latter, for example, involve $\gamma$ ray spectroscopy and the results of these measurements are expressed in terms of electron volts. The mass-energy conversion between the two scales requires the ratio $1 \mathrm{u} / 1$ $\mathrm{eV}$, where

$$
1 \mathrm{u} / 1 \mathrm{eV}=4 c R_{\infty}\left(m_{p} / m_{e}\right) /\left[(2 e / h)\left(m_{p} / m_{u}\right) \alpha^{2}\right] .
$$

We see from this expression that if the energies are expressed in terms of laboratory volts which are maintained by using an adopted value for $2 e / h$ the above reduces to [8]

$1 \mathrm{u} / 1 \mathrm{eV}_{\mathrm{LAB}}=4 c R_{\infty}\left(m_{p} / m_{e}\right) /\left[(2 e / h)_{\mathrm{LAB}}\left(m_{p} / m_{u}\right) \alpha^{2}\right]$. 
The accuracy of this quantity depends essentially on the accuracy of $\alpha^{2}$.

\section{Mass Measurements and the Fundamental Physical Constants}

At the atomic level we mostly make measurements involving the inertial mass of a particle in vacuo, whereas present-day laboratory determinations are made in terms of gravitational mass, and the weighings take place in laboratory air. Measurements to date have shown that the equivalence of inertial and gravitational mass can be relied upon to the present levels of accuracy in mass measurement.

The present definition of the kilogram is deceptively simple [10] and thereby avoids mention of whether or not one should include the mass of any adsorbed gases, or moisture remaining from the lavage process. International comparisons of the various copies of the prototype kilogram are effected with an accuracy of a few parts in $10^{9}$. The precision of the mass comparisons is some ten times better than this; indeed, Speake and Quinn [15] are beginning to achieve $10^{-11}$ mass discrimination in their "fifth force" weighing experiment. Although we know the stability of one artifact mass standard with respect to another, we do not yet know how stable they are in an absolute sense to much better than a part in a million-so the reproducibility shown for the kilogram in Table $I$ is traditional rather than well established. The accuracy of mass calibrations offered routinely to customers by the various national standards laboratories is about 0.1 parts per million at one kilogram, but is reduced to a part per million for masses of about one gram. However, tenfold improved calibration accuracy is available in appropriate cases. There is a further loss of accuracy once one transfers from intercomparing platinum-iridium standards to stainless-steel standards (brass weights are calibrated to lesser accuracy).

The measurements of the fundamental constants have to take account of this loss of accuracy away from the kilogram, as also do realizations of the ampere, the volt, and the watt. Aside from the determination of the Faraday constant [16], which involved masses of about $5 \mathrm{~g}$, the determination of the high-field gyromagnetic ratio of the proton by Kibble and Hunt [17] used masses between 20 and $150 \mathrm{~g}$ for the Cotton balance, that of the Avogadro constant [18] used masses between $54 \mathrm{~g}$ to $1 \mathrm{~kg}$, and the watt realization [19] used $1 \mathrm{~kg}$. The absolute volt realization [20], involving the density of mercury, was effectively made at masses of 5 and $10 \mathrm{~kg}$, for these were the mass of mercury involved in the measurement of the density of mercury by Cook [21]. The other absolute measurements which are under development, and which are discussed elsewhere in this conference, are also made close to $1 \mathrm{~kg}$ [22].

\section{Can We Replace the Prototype Kilogram?}

To some extent the atomic mass unit does not have a real existence. One could, of course, maintain a ${ }^{12} \mathrm{C}$ atom
TABLE I

The Accuracies Associated with the Realization of atomic Mass, of Mass COMPaRISONS, AND OF THE VOLT

\begin{tabular}{|c|c|c|c|}
\hline Accuracy of usage & amu & $\begin{array}{c}\text { Unt t } \\
\text { kilogram }\end{array}$ & volt \\
\hline $\begin{array}{l}\text { Fractional accuracy of } \\
\text { realisation in SI units (*) }\end{array}$ & $6 \times 10^{-7}$ & $\because 10^{-9}$ & $\sim 10^{-7}$ \\
\hline $\begin{array}{l}\text { Fractional accuracy with } \\
\text { which maintained }\end{array}$ & $\leqslant 10^{-8}$ & $<10^{-8}$ & $\sim 10^{-8}$ \\
\hline $\begin{array}{l}\text { Accuracy of calibration, } \\
\text { dissemination, or use }\end{array}$ & $10^{-9}-10^{-8}$ & $10^{-7}-10^{-8}$ & $\sim 10^{-7}$ \\
\hline $\begin{array}{l}\text { Present best fractional } \\
\text { sensitivity }\end{array}$ & $\sim 10^{-9}$ & $\sim 10^{-12}$ & $\sim 10^{-15}-10^{-10} \sigma_{(+)}$ \\
\hline
\end{tabular}

$(*)$ at the time of the 1986 CODATA evaluation.

$(+)$ in superconducting circuits.

in an atom trap, but there is no need to do so, for the mass is readily transferred to other atomic mass standards. For example, one can easily transfer from the ${ }^{12} \mathrm{C}$ atom to the ${ }^{12} \mathrm{C}^{+}$ion. The improved accuracy of the realization of the atomic mass unit in terms of the kilogram is shown in Fig. 1. It is seen that until recently our knowledge of $m_{p} / m_{u}$ had been making more rapid progress than our knowledge of $m_{u}$ in terms of the kilogram, so that the gap had been widening. Progress with the evaluation of $m_{p} / m_{u}$ has slowed since the doublet measurements of Lincoln Smith in the early 1970's, and the gap has closed. The nuclidic mass of the proton is now thought to be $1.007276468(7)$ $\mathrm{u}$ (as a result of the latest, interim, mass adjustment [23]), in place of $1.007276470(11) \mathrm{u}$. There is a natural pace to metrology, which is related to that of science and technology, and one can see from the figure that if the steady state in our knowledge of $m_{u}$ thus far were extrapolated, one would expect to reach the $10^{-8}$ level of accuracy within 25 years; consequently a more rapid rate of progress must be found if we wish to hasten the replacement of the kilogram! Meanwhile, ion traps have already been used for measurements of the atomic masses of particles which are far from stability [24], so it is likely that progress in relative atomic mass measurements will continue for at least a few more decimal places, possibly to $\sim 10^{-12}$ fractional uncertainties within 25 years or so-no doubt with exciting results.

The first generation of measurements of the Avogadro constant pioneered by Deslattes et al. at the NBS, and more recently by Seyfried et al. at the PTB, achieved part per million accuracy and the second generation in progress at the NBS, PTB, and elsewhere might achieve 0.1 parts per million. This would still leave the third generation to take us to fractional accuracies of $10^{-8}$ and beyond. Formidable problems of silicon sample fabrication and measurement would have to be solved before this accuracy could be achieved.

Ideally, the replacement of the present definition of the kilogram by an atomic one should permit a further improvement in measurement accuracy (both for masses that 


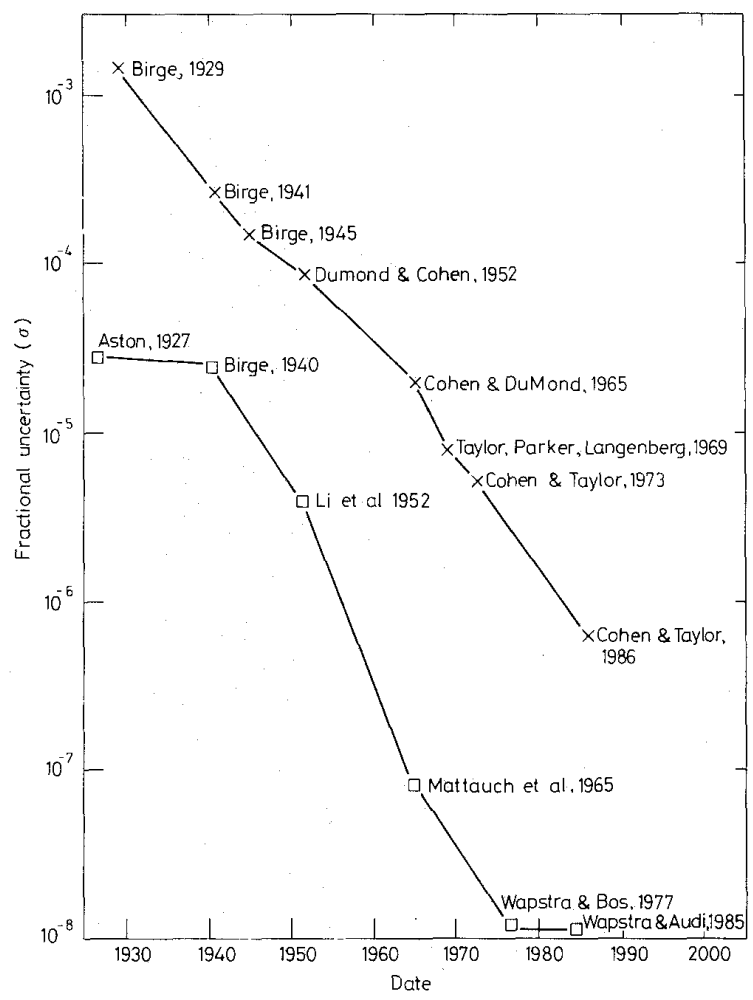

Fig. 1. The improved accuracy since 1929 of our knowledge of the atomic mass unit and the relative atomic mass of the proton.

are close to the kilogram and those at atomic mass levels) by at least an order of magnitude before it too became obsolete. In this regard there is an important lesson to be remembered from the krypton-86 definition of the meter. The concept took more than a century to realize practically, yet endured for only 23 years [25]. We have arrived at a very silly situation indeed if school children have to learn a new definition each time that we fine-tune our metrology in the $n$th decimal place!

For the present the realizations of the electrical units play a pivotal role in determining the accuracies with which we know the fundamental physical constants. Thus [19], the realization of the ampere determines $e$, the ohm $h / e^{2}$, the volt $2 e / h$, and the watt determines $h$. It is tempting, therefore, to replace the kilogram and the ampere with the Josephson-effect volt as a base unit, with the impedance of free space retained as $\mu_{0} c$-the latter would have pleased Giorgi! The precision of the present usage of the atomic mass unit, the kilogram, and the volt is shown in Table I. It appears from this that, unless we are content to transfer the imprecision of the volt to the $\mathrm{ki}$ logram, a change of usage would be inappropriate at present. It is possible that some other quantum phenomena might become measurable with adequate accuracymuch as the quantized Hall resistance arrived less than ten years ago. The Josephson effects in liquid helium, for example, show us how a quantum force effect could arrive which might be refinable as a quantum force standard in the future; these particular effects, though, have been discovered, "undiscovered," and rediscovered [26]. Such questions would have to be debated in depth by the global scientific and technological community before being put into effect and the electrical units would be used to establish and monitor the stability of the kilogram ahead of such a decision.

The problem is not one of finding an atomic mass unit, for we already have one, but rather in making the conversion from an atomic scale to the macroscopic world with adequate accuracy, so that the prototype kilogram may be replaced. The kilogram is of a convenient size for everyday use in commerce and technology and is roughly central between the masses of atomic particles and the mass of the universe. It is, therefore, likely that we would wish to retain the present size for our mass unit-even if the definition were to be changed in favor of one based on some type of atomic quantity.

The atomic mass unit would be known with slightly improved accuracy if we chose to change the status of one of the electrical units, and transferred their imprecision to the kilogram by making the latter a derived unit. Even if such a change were accepted a separate atomic mass unit would still be required. This situation is likely to continue for the next 15 to 25 years.

\section{REFERENCES}

[1] M. Kochsiek, "The mass unit 'kilogram,' precision measurement of mass, attainable uncertainties and possibilities of a new definition." Precision Measurements and Fundamental Constants, vol. II, B. N. Taylor and W. D. Phillips, Eds. (NBS Spec. Publ. 617). Washington: Government Printing Office, 1984, pp. 427-435.

[2] Le Système International d'Unités (SI), 5th ed. Sèvres: Bureau International des Poids et Mesures, 1985.

[3] H. G. J. Moseley, "The high frequency spectrum of the elements," Phil. Mag., vol. 26, pp. 1024-1034, Dec. 1913.

[4] F. D. Rossini, Fundamental Measures and Constants for Science and Technology. Cleveland: CRC Press, 1973.

[5] . F. W. Aston, "The unit of atomic weight," Rep. Brit. Assoc., pp. 333-335, Sept. 1931.

[6] D. H. Whiffen, "The unified atomic mass unit," J. Roy. Inst. Chem. (GB), vol. 84, 133-136, Apr. 1960.

[7] H. F. Dixon, "The Dalton and the atomic mass unit," Trends in Biochem. Sc., vol. 8, pp. 49-52, Feb. 1983.

[8] N. N. Greenwood and H. S. Peiser, "Table of atomic weights to four significant figures," Chem. Int., vol. 10, 94-97, May, 1988.

[9] A. H. Wapstra and G. Audi, "The 1983 atomic mass table." Nuct. Phys. A, vol. 432, nos. 1-4, pp. 231-234, Jan. 1985; A. H. Wapstra and K. Bos, At. and Nucl. Data Tab. vol. 19, pp. 177-214. Mar. 1987; and "The 1977 atomic mass evaluation IV: Evaluation of input values; adjustment procedures," At. Nucl. Data Tables, vol. 20, pp. 1-26, July 1977.

[10] Quantities, Units and Symbols in Physical Chemistry. Oxford: Blackwell Scientific Publications, 1988.

[11] E. R. Cohen and B. N. Taylor, "The 1986 adjustment of the fundamental physical constants,' Rev. Mod. Phys., vol. 59, 1121-1148, Oct. 1987.

[12] L. G. Smith, "Measurements of six light masses," Phvs. Rev. C(USA) vol. 4, pp. 22-31, Jan. 1971; and L. G. Smith and A. H. Wapstra, "Masses of isotopes of H, He, C, N, O and F," Phys. Rev. C(USA), vol. 11, pp. 1392-1400, Apr. 1975.

[13] W. H. Johnson, Jr., "The measurement of atomic masses by mass spectroscopic methods and a role for atomic masses in the determination of the fundamental constants," in Precision Measurements and Fundamental Constants, vol. II, B. N. Taylor and W. D. Phillips. Eds. Washington: Government Printing Office, pp. 335-344; J. L. Benson and W. H. Johnson, Jr., "Isotopic masses of hydrogen, chlo- 
rine, barium, cesium and neodymium," Phys, Rev., vol. 141, pp. 1112-1122, Jan. 1966

[14] E. R. Cohen and A. H. Wapstra, "Recommended treatment of precision measurements related to nuclear energy levels," Nucl. Instrum. Methods, vol. 211, pp. 153-157, June 1983.

[15] C. C. Speake and T. J. Quinn, "A measurement of the isotopic spin component of the fifth force by weighing," IEEE Trans. Instrum. Meas., pp. 189-195, this issue.

[16] V. E. Bower and R. Davis, "The electrochemical equivalent of silver-A value of the Faraday," J. Res. Natl. Bur. Stand., vol. 85, $175-191,1980$.

[17] B. P. Kibble and J. Hunt, "A measurement of the gyromagnetic ratio of the proton in a strong magnetic field," Metrologia, vol. 15, pp. 5$30,1979$.

[18] R. D. Deslattes et al., "Avogado constant-Correction to an earlier report,” Phys. Rev. Lett., vol. 46, pp. 898-900, Dec. 1986.

[19] B. W. Petley, A. Hartland and B. P. Kibble, "A measurement of the Planck constant," Nature, vol. 327, pp. 605-606, Jan. 1987.

[20] G. J. Sloggett, W. K. Clothier, and B. W. Ricketts, "Determination of $2 e / h$ and $h / e^{2}$ in SI units," Phys. Rev. Lett., vol. 57, pp. 32373244, Dec. 1986.

[21] A. H. Cook, "Precision measurement of the density of mercury at $20^{\circ} \mathrm{C}$," Phil. Trans. Roy. Soc. London, vol. 254, pp. 125-138, Nov. 1961.

[22] F. Shiota et al., "Improvement of the superconducting magnetic levitation system for the determination of the magnetic flux quantum,' pp. 225-228, this issue.

[23] A. H. Wapstra and G. Audi, "Mid-stream mass adjustment," Nucl. Phys. A, to be published.

[24] F. Kern, et al., "Ion trap mass measurements," in Nuclei far from Stability, I. A. Towner. Ed. (AIP Conference Proc. 164). New York: American Institute of Physics, 1988, pp. 20-29.

[25] B. W. Petley, "On the new definition of the meter," Nature, vol. 303 , pp. 373-376, June 1983.

[26] O. Avenel and E. Varoquaux, "Observation of singly quantized dissipation events obeying the Josephson frequency relation in the critical flow of superfluid ${ }^{4} \mathrm{He}$ through an aperture," Phys. Rev. Lett. vol. 55, pp. 2704-2707, Dec. 1985. 